\title{
Late Bone Metastasis of Histologically Bland Struma Ovarii: The Unpredictability of Its Biologic Behavior
}

\author{
Sun-Ju Oh · Minjung Jung · Young-Ok Kim \\ Department of Pathology, Kosin University Gospel Hospital, Busan, Korea
}

Struma ovarii (SO) is a subtype of ovarian teratoma that contains mostly thyroid tissue comprising more than $50 \%$ of the tumor volume. ${ }^{1}$ It is the most common type of monodermal teratoma of the ovary, accounting for approximately $2.7 \%$ of all teratomas. ${ }^{1}$ Most cases of typical SO are benign with exceptional cases showing malignant features of papillary carcinoma, vascular invasion, or invasion into surrounding tissue. However, there are rare cases of histologically benign $\mathrm{SO}$ with malignant biological behavior, variously designated as peritoneal strumosis, malignant SO, metastatic SO, or highly differentiated follicular carinomas. ${ }^{2,3}$ Here, we report on a patient who developed a vertebral metastasis ten years after oophorectomy for $\mathrm{SO}$ that finally proved to be malignant.

\section{CASE REPORT}

A 60-year-old woman presented with back pain. Magnetic resonance imaging revealed a destructive bone mass at the thoracic 12 level of the spine (Fig. 1A). Overall image analysis suggested a metastasis, but the origin could not be detected on a positron emission tomography-computed tomography (PET-CT) scan. Needle biopsy was performed on the spinal lesion, and microscopically it revealed thyroid follicles with colloids resembling normal thyroid tissue (Fig. 1B, C). Immunohistochemically, these cells were diffusely positive for thyroid transcription factor-1 and thyroglobulin (Fig. 1D), suggesting thyroid as origin of the

\section{Corresponding Author}

Young-OK Kim, MD

Department of Pathology, Kosin University Gospel Hospital, 262 Gamcheon-ro,

Seo-gu, Busan 602-702, Korea

Tel: +82-51-990-6744, Fax: +82-51-990-3080,

E-mail: 10highpowerfield@gmail.com

Received: April 1, 2015 Revised: April 22, 2015

Accepted: April 27, 2015 tumor. Any evidence of nuclear atypia suspicious for papillary carcinoma was not detected throughout the lesion, which was consequently diagnosed as metastatic follicular carcinoma of thyroid.

Subsequent clinical evaluation revealed a high serum thyroglobulin level of $1,277 \mathrm{ng} / \mathrm{mL}$ (normal range, 1.4 to $78.0 \mathrm{ng} / \mathrm{mL}$ ). However, ultrasonogram and computed tomography showed no distinct mass in the thyroid gland. The patient's past medical history revealed that she underwent total hysterectomy and bilateral salpingo-oophorectomy for a left ovarian cyst 12 years ago that was initially diagnosed as SO. Follow-up of the patient included periodic ultrasonograms and measurement of serum cancer antigen 125 levels, all of which had been normal since the surgery. A suspicion for metastasis originating from the ovarian tumor was raised, and the initial slides were reevaluated.

Upon review of the pathology report and slides, the ovarian cyst was a 12-cm-sized multilocular mass with a smooth outer surface. Microscopic examination showed benign looking thyroid tissue characterized by round follicles, most of which were dilated, but some follicles were small and others were medium sized. Many follicles contained intraluminal colloids, and they were lined by cuboidal epithelial cells with moderate amounts of cytoplasm (Fig. 2A). However, a complete examination revealed a focus of 0.5 -cm-sized closely packed microfollicles with nuclear irregularity, overlapping, and vague clearing (Fig. 2B, C). These features resembled a follicular variant of papillary carcinoma (FVPC), but were insufficient to establish a definite diagnosis of FVPC. Other characteristics suspicious for malignancy such as vascular or ovarian capsular invasion were not noted. No other teratomatous components were identified. A possibility of unusual metastasis from $\mathrm{SO}$ was suggested for the spinal lesion.

Subsequent total thyroidectomy and spondylectomy for the spinal lesion were conducted. The resected thyroid gland showed no evidence of neoplasm, as expected, and the spinal lesion ex- 

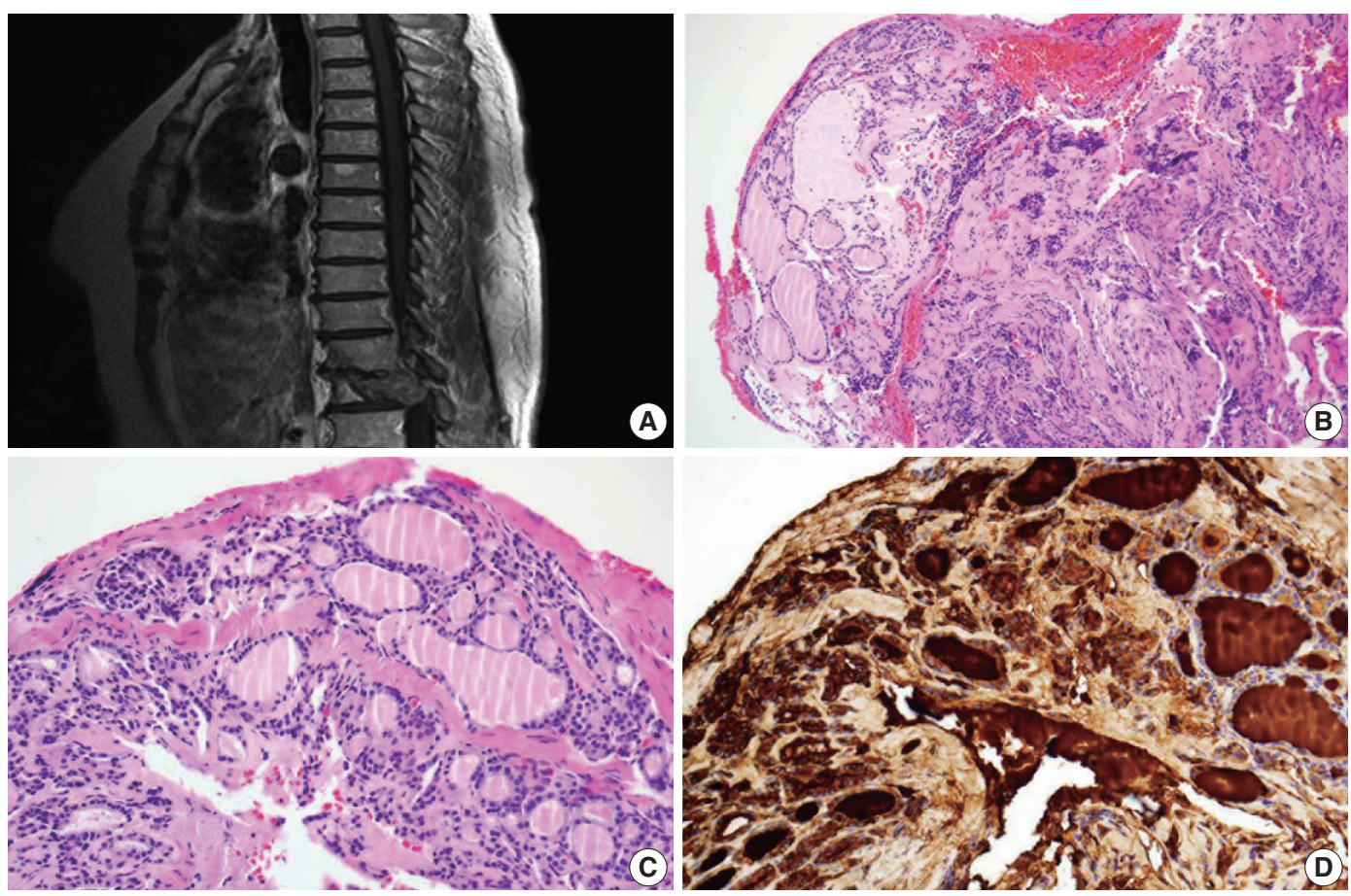

Fig. 1. (A) Sagittal magnetic resonance imaging showing an ill-defined lytic mass involving the thoracic 12 level. The mass destructs the vertebral body and spinal canal extending to the pedicle. $(B, C)$ Needle biopsy of the spinal lesion reveals benign-looking thyroid follicles. (D) These cells are positive for thyroglobulin on immunohistochemical stain, supporting thyroid origin.
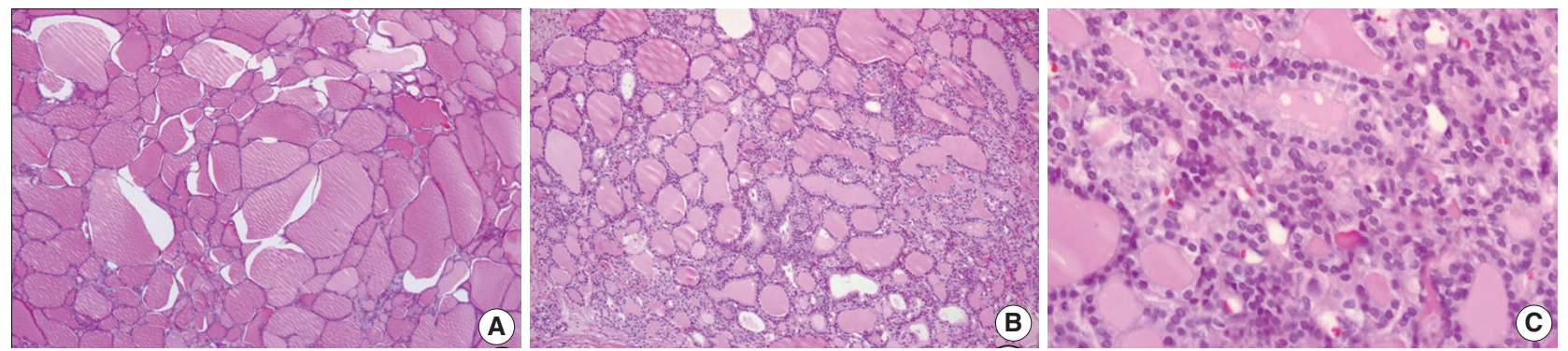

Fig. 2. Histological features of struma ovarii. Most of the tumor consist of dilated follicles reminiscent of nodular goiter (A) while a small part of the tumor shows densely packed microfollicles (B) with mild nuclear irregularity, overlapping, and vague clearing (C).

hibited the same histological features as the needle biopsy. The patient subsequently received radioactive iodine (RAI) treatment, but her PET-CT scan on the last follow-up showed multiple bone metastases in the scapula, rib, sternum, and pelvic bones.

\section{DISCUSSION}

The most common thyroid-type carcinomas originating in SO are papillary and follicular carcinomas. The diagnostic criteria for cases of papillary carcinoma are similar to those described for the cervical thyroid gland and are based primarily on nuclear and architectural features. ${ }^{4}$ These features are almost invariable and of no clinical importance. With respect to follicular carcinoma, invasion into the surrounding ovarian tissue, vascular invasion, or metastasis is evidence of malignancy. ${ }^{5}$

However, $\mathrm{SO}$ can show a wide spectrum of histological features that should not prompt a diagnosis of malignancy. These include the presence of areas resembling follicular adenoma, foci with papillary architecture, and nuclear clearing. SO with these features have a benign clinical course and these tumors are designated "proliferative SO." These types of tumors generally do not behave in a malignant fashion. ${ }^{4}$ However, this claim was denied later when adenomatous proliferative $\mathrm{SO}$ also can show the propensity for recurrence or metastasis. ${ }^{6}$

It is difficult to predict the metastatic potential of $\mathrm{SO}$ at initial diagnosis. Even SO that histologically resembles non-neo- 
plastic thyroid tissue is sometimes associated with recurrence or extra-ovarian metastasis, which have previously been designated as peritoneal strumosis, malignant $\mathrm{SO}$, metastatic $\mathrm{SO}$, or related terms. ${ }^{3}$ Roth and Karseladze ${ }^{2}$ described three cases of $\mathrm{SO}$ with extra-ovarian dissemination having an innocuous appearance resembling that of a colloid or nodular goiter. They designated this entity as "highly differentiated follicular carcinoma of ovarian origin (HDFCO)." The diagnosis of HDFCO characteristically cannot be made until extra-ovarian dissemination is detected because of its non-neoplastic appearance. They also compared cases of HDFCO with those of typical thyroid-type carcinomas of ovarian origin with peritoneal involvement. They found that biological behavior is not different in the point that the prognosis seems to be favorable.

In a study of 27 cases of biologically malignant $\mathrm{SO}$ with extraovarian dissemination or metastasis, Shaco-Levy et al. ${ }^{6}$ found that histologically malignant, adenomatous, or even normal primary tumors can show biologically malignant behavior. The authors found no independent factor to predict its biological behavior. However, severe fibrous adhesions, larger amounts of peritoneal fluid $(\geq 1 \mathrm{~L})$, and larger size of the stromal component $(\geq 12 \mathrm{~cm})$ have some predictive value. In the following year, the authors analyzed in more detail the natural history of biologically aggressive tumors in a study of patients with more rapid disease progression. ${ }^{7}$ Factors predictive of a potentially more aggressive clinical course are large tumor size (>10 cm), more than $80 \%$ stromal tissue, extensive papillary carcinoma histology, more than five mitoses per 10 high-power field, and marked cytological atypia. Our case showed no adverse histological or clinical factors other than the tumor size of $12 \mathrm{~cm}$ according to their criteria. However, others have contradicted that the size of the stromal component is not relevant to the metastatic or recurrence potential and no histologic features seem to correlate with a propensity for an adverse clinical outcome. ${ }^{8}$

When no independent factor predicts biologically malignant potential of SO, controversy regarding the extent of pelvic resection and the management of the thyroid gland can arise with respect to the treatment plan. Marti et al. ${ }^{9}$ suggested that pelvic surgery alone may be sufficient initial therapy for thyroid-type carcinoma confined to the ovary, whereas prophylactic total thyroidectomy with RAI may be reserved for patients with extraovarian spread or distant metastasis. However, there are no present series and data from the literature allowing the determination of risk stratification for patients with SO confined to the ovary at presentation. Because recurrence may occur after more than a decade following diagnosis, as in the present case, long- term follow-up is indicated with regular thyroglobulin measurements to detect metastasis.

In summary, we report a patient with a vertebral metastasis 10 years after diagnosis of histologically benign SO. On the basis of our experience with this patient and others in the literature, no determinants can predict its biologically malignant potential. Pathologists are advised to report in detail on tumor size, cytologic atypia, mitosis, and other histological factors affecting the adverse outcome when encountering large SO consisting solely of thyroid tissue as in our case. Long-term follow-up with regular thyroglobulin measurements should be warranted for any evidence of metastasis or recurrence.

\section{Conflicts of Interest}

No potential conflict of interest relevant to this article was reported.

\section{REFERENCES}

1. Kurman RJ, Carcangiu ML, Herrington CS, Young RH. WHO classification of tumours of female reproductive organs. 4th ed. Lyon: IARC Press, 2014.

2. Roth LM, Karseladze AI. Highly differentiated follicular carcinoma arising from struma ovarii: a report of 3 cases, a review of the literature, and a reassessment of so-called peritoneal strumosis. Int J Gynecol Pathol 2008; 27: 213-22.

3. Karseladze AI, Kulinitch SI. Peritoneal strumosis. Pathol Res Pract 1994; 190: 1082-5.

4. Devaney K, Snyder R, Norris HJ, Tavassoli FA. Proliferative and histologically malignant struma ovarii: a clinicopathologic study of 54 cases. Int J Gynecol Pathol 1993; 12: 333-43.

5. Rosenblum NG, LiVolsi VA, Edmonds PR, Mikuta JJ. Malignant struma ovarii. Gynecol Oncol 1989; 32: 224-7.

6. Shaco-Levy R, Bean SM, Bentley RC, Robboy SJ. Natural history of biologically malignant struma ovarii: analysis of 27 cases with extraovarian spread. Int J Gynecol Pathol 2010; 29: 212-27.

7. Robboy SJ, Shaco-Levy R, Peng RY, et al. Malignant struma ovarii: an analysis of 88 cases, including 27 with extraovarian spread. Int J Gynecol Pathol 2009; 28: 405-22.

8. Garg K, Soslow RA, Rivera M, Tuttle MR, Ghossein RA. Histologically bland "extremely well differentiated" thyroid carcinomas arising in struma ovarii can recur and metastasize. Int J Gynecol Pathol 2009; 28: 222-30.

9. Marti JL, Clark VE, Harper H, Chhieng DC, Sosa JA, Roman SA. Optimal surgical management of well-differentiated thyroid cancer arising in struma ovarii: a series of 4 patients and a review of 53 reported cases. Thyroid 2012; 22: 400-6. 